\title{
Particle density fluctuations
}

\author{
Bedangadas Mohanty for the WA98 Collaboration *
}

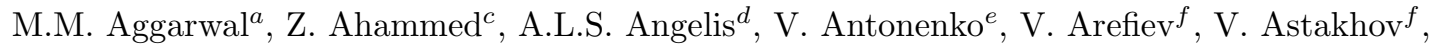
V. Avdeitchikov ${ }^{f}$, T.C. Awes ${ }^{g}$, P.V.K.S. Baba ${ }^{h}$, S.K. Badyal ${ }^{h}$, S. Bathe ${ }^{i}$, B. Batiounia ${ }^{f}$, T. Bernier $^{j}$, K.B. Bhalla ${ }^{b}$, V.S. Bhatia ${ }^{a}$, C. Blume ${ }^{i}$, D. Bucher ${ }^{i}$, H. Büsching ${ }^{i}$, L. Carlen ${ }^{m}$, S. Chattopadhyay ${ }^{c}$, A.C. Das ${ }^{c}$, M.P. Decowski ${ }^{l}$, P. Donni ${ }^{d}$, A.K. Dubey ${ }^{s}$, M.R. Dutta Majumdar ${ }^{c}$, K. Enosawa ${ }^{n}$, S. Fokin $^{e}$, V. Frolov ${ }^{f}$, M.S. Ganti ${ }^{c}$, S. Garpman ${ }^{m}$, O. Gavrishchuk ${ }^{f}$, F.J.M. Geurts ${ }^{l}$, R. Glasow ${ }^{i}$, B. Guskov ${ }^{f}$, H.A. Gustafsson ${ }^{m}$, H.H. Gutbrod ${ }^{j}$, I. Hrivnacova ${ }^{o}$, M. Ippolitov ${ }^{e}$, H. Kalechofsky ${ }^{d}$, R. Kamermans ${ }^{l}$, K. Karadjev ${ }^{e}$, K. Karpio ${ }^{q}$, B.W. Kolb ${ }^{k}$, I. Kosarev ${ }^{f}$, I. Koutcheryaev ${ }^{e}$, A. Kugler ${ }^{o}$, P. Kulinich ${ }^{r}$, M. Kurata ${ }^{n}$, A. Lebedev ${ }^{e}$, H. Löhner ${ }^{p}$, D.P. Mahapatra ${ }^{s}$, V. Manko ${ }^{e}$, M. Martin ${ }^{d}$, Y. Miake ${ }^{n}$, G.C. Mishra ${ }^{s}$, B. Mohanty ${ }^{c}$, D. Morrison ${ }^{t}$, D.S. Mukhopadhyay ${ }^{c}$, H. Naef ${ }^{d}$, B.K. Nandi ${ }^{s}$, S.K. Nayak ${ }^{j}$, T.K. Nayak $^{c}$, A. Nianine ${ }^{e}$, V. Nikitine ${ }^{f}$, S. Nikolaev ${ }^{e}$, S. Nishimura ${ }^{n}$, P. Nomokov ${ }^{f}$, J. Nystrand $^{m}$, A. Oskarsson ${ }^{m}$, I. Otterlund ${ }^{m}$, S.C. Phatak ${ }^{s}$, S. Pavliouk ${ }^{f}$, T. Peitzmann ${ }^{i}$, V. Petracek ${ }^{o}$ F. Plasil ${ }^{g}$, M.L. Purschke ${ }^{k}$,J. Rak ${ }^{o}$, R. Raniwala ${ }^{b}$, S. Raniwala ${ }^{b}$, N.K. Rao ${ }^{h}$, F. Retiere ${ }^{j}$, K. Reygers $^{i}$, G. Roland $^{r}$, L. Rosselet $^{d}$, I. Roufanov ${ }^{f}$, J.M. Rubio ${ }^{d}$, S.S. Sambyal ${ }^{h}$, R. Santo ${ }^{i}$, S. Sato ${ }^{n}$, H. Schlagheck ${ }^{i}$, H.-R. Schmidt ${ }^{k}$, Y. Schutz ${ }^{j}$, G. Shabratova ${ }^{f}$, I. Sibiriak ${ }^{e}$, T. Siemiarczuk ${ }^{q}$, B.C. Sinha ${ }^{c}$, N. Slavine $^{f}$, K. Söderström ${ }^{m}$, G. Sood ${ }^{a}$, S.P. Sørensen ${ }^{t}$, P. Stankus ${ }^{g}$, G. Stefanek ${ }^{q}$, P. Steinberg ${ }^{r}$, E. Stenlund ${ }^{m}$, M. Sumbera ${ }^{o}$, T. Svensson ${ }^{m}$, M.D. Trivedi ${ }^{c}$, A. Tsvetkov ${ }^{e}$, L. Tykarski $^{q}$, J. Urbahn $^{k}$, N.v. Eijndhoven ${ }^{l}$, G.J.v. Nieuwenhuizen ${ }^{r}$, A. Vinogradov ${ }^{e}$, Y.P. Viyogi ${ }^{c}$, A. Vodopianov $^{f}$, S. Vörös ${ }^{d}$, B. Wyslouch ${ }^{r}$, and G.R. Young $g$

${ }^{a}$ Univ. of Panjab (India), ${ }^{b}$ Univ. of Rajasthan (India), ${ }^{c}$ VECC, Calcutta (India), ${ }^{d}$ Univ. of Geneva (Switzerland), ${ }^{e}$ Kurchatov (Russia), ${ }^{f}$ JINR, Dubna (Russia), ${ }^{g}$ ORNL, Oak Ridge (USA), ${ }^{h}$ Univ. of Jammu (India), ${ }^{i}$ Univ. of Münster (Germany), ${ }^{j}$ SUBATECH, Nantes (France), ${ }^{k}$ GSI, Darmstadt (Germany), ${ }^{l}$ NIKHEF, Utrecht (The Netherlands), ${ }^{m}$ Univ. of Lund (Sweden), ${ }^{n}$ Univ. of Tsukuba (Japan), ${ }^{o}$ NPI, Rez (Czech Rep.), ${ }^{p} \mathrm{KVI}$, Groningen (The Netherlands), ${ }^{q}$ INS, Warsaw (Poland), ${ }^{r}$ MIT, Cambridge (USA), ${ }^{s}$ OP, Bhubaneswar (India), ${ }^{t}$ Univ. of Tennessee (USA)

Event-by-event fluctuations in the multiplicities of charged particles and photons at SPS energies are discussed. Fluctuations are studied by controlling the centrality of the reaction and rapidity acceptance of the detectors. Results are also presented on the eventby-event study of correlations between the multiplicity of charged particles and photons to search for DCC-like signals.

\section{Introduction}

At high temperatures or high baryon number density, Quantum Chromo-Dynamics (QCD) describes a world of weakly interacting quarks and gluons very different from the hadronic world in which we live. This suggests the possibility of a phase transition as the temperature or density is increased in which there is a transition from a state of

\footnotetext{
*Presented at Quark Matter 2002, Nantes, France. email : bmohanty@veccal.ernet.in
} 
matter where quarks are confined inside hadrons to one where quarks are free to move around (deconfined) within a large volume - the quark gluon plasma (QGP). This can be addressed through experimental studies involving relativistic heavy-ion collisions. Experimental searches have focused on isolating signatures of two types of phase transitions which might occur in extremely hot and/or dense nuclear matter. One is related to the deconfinement of quarks while the other is related to chiral symmetry restoration. There is also an interesting possibility of the existence of a tri-critical point in the phase diagram [1], where the transition changes from first to second order. At the tri-critical point, one would observe singularities in several thermodynamical variables, such as the specific heat and matter compressibility. The thermodynamical variables are related to event-byevent fluctuations in experimental observables like particle multiplicity, transverse energy, and mean transverse momentum. For example, the total heat capacity is related to transverse momentum fluctuations and matter compressibility to multiplicity fluctuations [2,3]. Hence the existence of the tri-critical point can be probed in an experiment by varying the control parameters of the reaction (impact parameter, rapidity acceptance, beam energy, and system size) and studying the event-by-event fluctuations in the global observables. It is also believed that in high energy heavy-ion collisions there is a possibility of creating a chiral symmetry restored phase. One of the possible interesting consequences of chiral symmetry restoration, is the formation of disoriented chiral condensates (DCC) [4]. The detection and study of a DCC state is expected to provide valuable information about the chiral phase transition and vacuum structure of strong interactions. The probability distribution of the neutral pion fraction in a DCC domain follows the relation :

$$
P(f)=1 / 2 \sqrt{f} \quad \text { where } \quad f=N_{\pi^{0}} / N_{\pi} .
$$

Since the majority of charged particles consist of charged pions and majority of photons originate from $\pi^{0}$ decays, DCC formation in a given domain would be associated with large correlated event-by-event fluctuations in the multiplicities of charged particles and photons. Here we present the experimental results of multiplicity fluctuations and correlations in relativistic heavy-ion $(\mathrm{Pb}+\mathrm{Pb})$ collisions as measured by the WA98 experiment [5] at the CERN SPS.

\section{Multiplicity fluctuations}

Recently, much theoretical interest has been generated on the subject of event-by-event fluctuations, primarily motivated by the nearly perfect Gaussian distributions of several observables $\left(N_{\gamma}, N_{c h}, E_{T}\right.$, and $\left.<p_{T}>\right)$ for a fixed centrality bin [3],6]. One may define the relative fluctuation as,

$$
\omega_{X}=\frac{\sigma_{X}^{2}}{<X>}
$$

where $\sigma_{X}^{2}$ is the variance of the distribution. The fluctuation is then studied by varying the control parameters of the reaction, such as the centrality and rapidity acceptance.

The WA98 experiment at the CERN SPS has carried out a detailed analysis of photon and charged particle multiplicity [6] fluctuations. The photon multiplicity is measured using the photon multiplicity detector (PMD) [7] which has the pseudo-rapidity coverage from 2.9 to 4.2 . The charged particle multiplicity is obtained from the silicon pad 
multiplicity detector (SPMD) [8] with pseudo-rapidity coverage from 2.35 to 3.75 . The centrality of the reaction is defined through the measurement of transverse energy $\left(E_{T}\right)$ with the mid-rapidity calorimeter (MIRAC) having a pseudo-rapidity coverage from 3.5 to 5.5 .

\subsection{Centrality dependence of multiplicity fluctuations}

It is very important to control the centrality selection carefully in fluctuation studies so that the impact parameter fluctuations are kept to a minimum and the distributions are good Gaussians. With this in mind we have used narrow centrality selections with bin widths of $2 \%$ in cross section, as determined from the measured total transverse energy, such as, $0-2 \%, 2-4 \%, 4-6 \%, 6-8 \%$, etc. The fluctuations calculated for these centrality bins using Eqn. 1 are plotted in Fig. 1 as a function of the number of participants. The results from data for both charged particles and photons are compared to those obtained from the VENUS event generator and a simple participant model [6]. In the participant model, where the total particle multiplicity in an event is the sum of the number of particles produced by each participant, the multiplicity fluctuations, $\omega_{N}$, can be expressed as

$$
\omega_{N}=\omega_{n}+\langle n\rangle \omega_{N_{\text {part }}}
$$

The value of $\omega_{n}$, the fluctuation in the number of particles falling within the detector acceptance $(n)$ produced per participant can be obtained from nucleon-nucleon data. The value of $\omega_{N_{\mathrm{part}}}$, the fluctuation in the number of participants, can be obtained from simulations. From Fig. 1 we observe that the measured fluctuations are reasonably well reproduced by statistical models.

\subsection{Acceptance dependence of multiplicity fluctuations}

Fluctuations in the particle multiplicity have also been studied by varying the rapidity acceptance. The results are shown in Fig. 2. One observes, contrary to naive expectation that the fluctuations should increase as the multiplicity decreases with decrease in acceptance, that instead the fluctuations decrease. This can be explained through a simple statistical model, based on the assumption that particles are accepted following a binomial distribution. The details of this model are given in Ref [9]. As per this model, the fluctuations $\left(\omega_{n}\right)$ in a small acceptance region are related to the fluctuations $\left(\omega_{m}\right)$ in a larger acceptance as

$$
\omega_{n}=1-f+f \omega_{m}
$$

where $f$ is the ratio of the average number of particles in the smaller acceptance to the average number of particles in the larger acceptance. The results from the model shown in Fig. 2 are found to be in reasonably good agreement with the data.

\section{3. $N_{\gamma}$ vs. $N_{c h}$ fluctuations}

The main motivation to study event-by-event fluctuations in the photon vs. charged particle multiplicity is to search for possible formation of DCCs. For this one would like a photon multiplicity detector and a charged particle multiplicity detector with as large a common coverage in $\eta-\phi$ phase space as possible. The main DCC search result from the SPS has come from the WA98 experiment 10,11]. First we discuss the results for top 5\% 

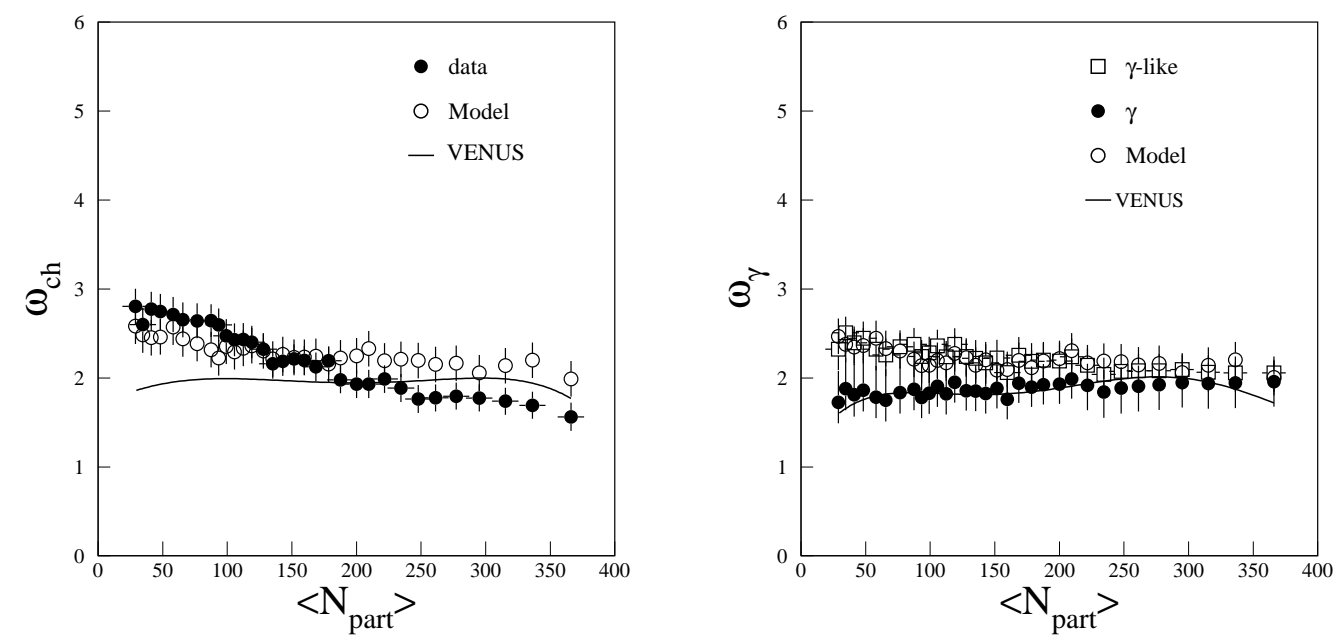

Figure 1. Multiplicity fluctuations of charged particles and photons for various centralities in a region of common coverage of the PMD and SPMD as a function of the number of participants. The $\gamma$ - like results correspond to fluctuations of the measured photons without correction for the photon counting efficiency or purity of the photon sample.
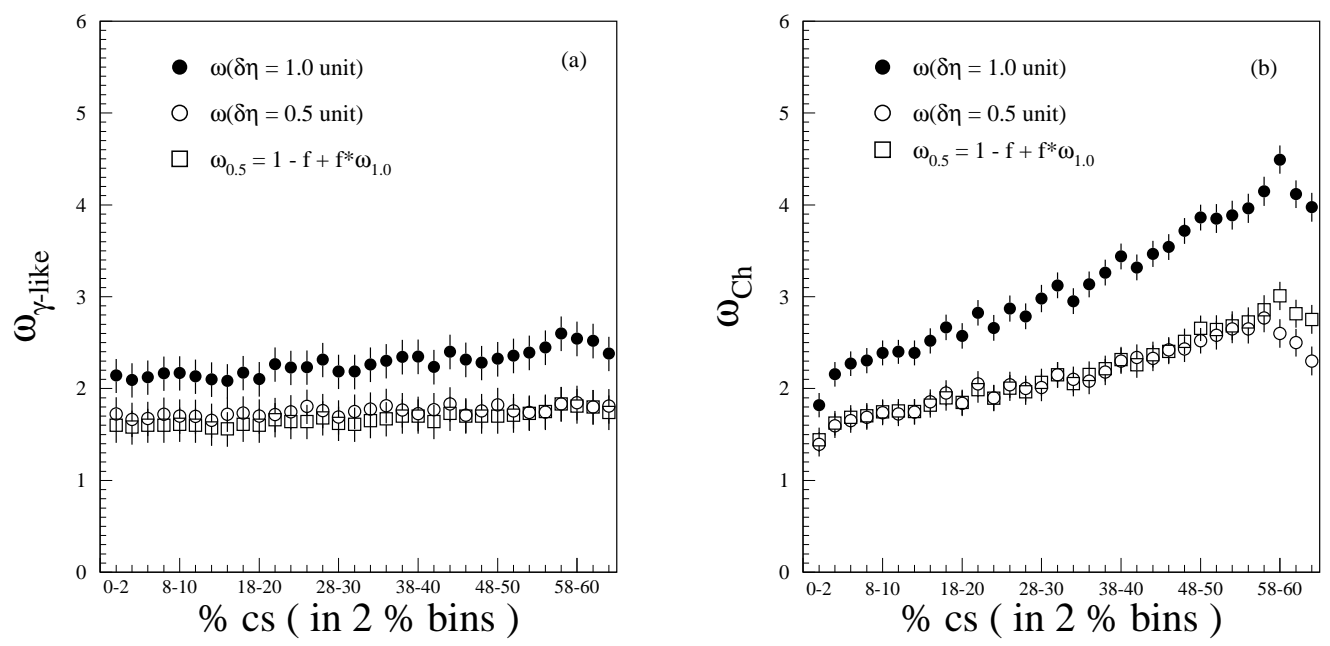

Figure 2. Multiplicity fluctuations of photons and charged particles for two $\eta$ acceptance selections. The open squares represent estimated fluctuation values in 0.5 unit of $\delta \eta$ from the observed fluctuations in 1.0 unit of $\delta \eta$. 
Table 1

Types of mixed events and the types of fluctuations probed.

\begin{tabular}{|cccc|}
\hline & PMD & SPMD & Type of fluctuation probed: \\
\hline M1 & Mix hits & Mix hits & Correlated + Individual \\
M2 & Unaltered hits & Unaltered hits & Correlated \\
M3- $\gamma$ & Unaltered hits & Mix hits & $N_{\gamma}$ only \\
M3-ch & Mix hits & Unaltered hits & $N_{\text {ch only }}$ \\
\hline
\end{tabular}

most central events. For the pseudo-rapidity and azimuthal region common to both PMD and SPMD used for this analysis, the average photon and charged particle multiplicity is 335 and 323, respectively. The experimental results are compared to simulated events and various types of mixed events to investigate possible DCC formation. The simulated events were generated by passing the VENUS output through a detector simulation package (GEANT), which incorporates the WA98 experimental setup. Four different types of mixed events were generated from the data with equivalent event sample sizes as for real events. The details of the construction of the mixed events can be found in Ref. [10]. In Table 1 we summarize the construction and the physics issue probed by each type of mixed event. A common analysis was carried out on the data, simulated, and mixed events to investigate the source of any observed fluctuations.

\section{1. $N_{\gamma}$ vs. $N_{c h}$ correlation analysis}

Analysis of the correlation between $N_{\gamma}$ and $N_{\text {ch }}$ is useful to search for DCC-type fluctuations. The details of this can be found in Ref. 88,10]. From the event-by-event correlation between $N_{\gamma \text {-like }}$ and $N_{\text {ch }}$ in various $\phi$-segments (obtained by dividing the $\phi$-space into 2 , 4,8 , and 16 bins) and a common correlation axis $(Z)$, one can obtain the closest distance $\left(D_{Z}\right)$ of the data points to the correlation axis. The correlation plot is shown in Fig. 3 . In order to compare the fluctuations for different $\phi$ bins on a similar footing, a scaled variable, $S_{Z}=D_{Z} / s\left(D_{Z}\right)$, is used, where $s\left(D_{Z}\right)$ represents the RMS deviation of the $D_{Z}$ distribution for VENUS events analyzed in the same manner. The width of the distribution of $S_{Z}$ represents the relative fluctuations of $N_{\gamma \text {-like }}$ and $N_{\mathrm{ch}}$ from the correlation axis at any given $\phi$ bin. Since the width of the $S_{Z}$ distribution quantifies the amount of fluctuation, the RMS deviations of these distributions for data are compared with those from mixed events and simulations in Fig. 4 .

The RMS deviations of the $S_{Z}$ distributions for mixed events and data agree for 1 bin in $\phi$ (by construction) and for 16 bins in $\phi$ within the quoted errors. However, for the other bins in $\phi$ one observes that results from M1 mixed events are lower than those from data. This indicates the presence of localised non-statistical fluctuations in the data. The source of the additional fluctuations is understood by the comparison to the M3-type of mixed events. Comparison shows that the excess fluctuations have contributions both from $N_{\gamma}$ and $N_{\mathrm{ch}}$. However the RMS deviations of the M2-type of mixed events closely follow those from data, suggesting the absence of event-by-event correlated DCC-like $N_{\gamma}$ vs. $N_{\text {ch }}$ fluctuations. 


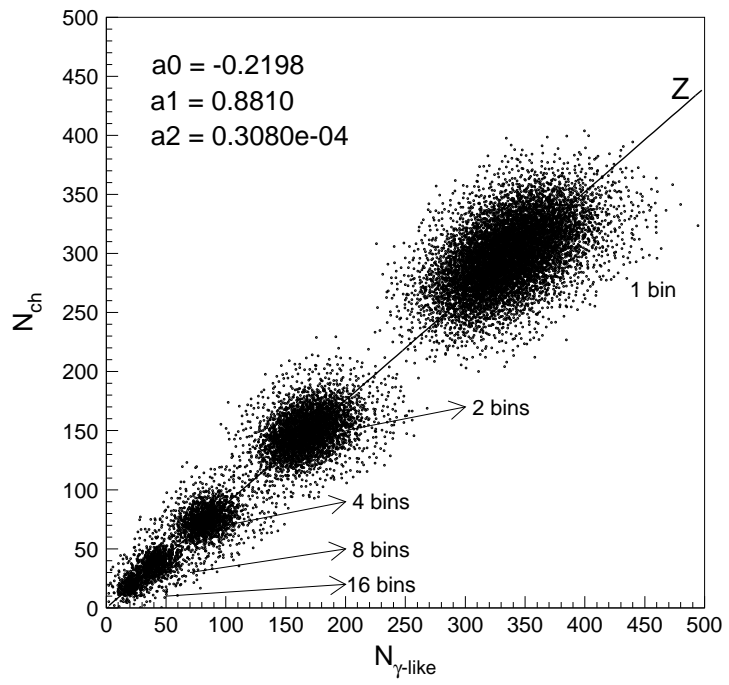

Figure 3. The event-by-event correlation between $N_{\mathrm{ch}}$ and $N_{\gamma-\text { like }}$ for the top $5 \%$ centrality class. Overlaid on the plot is the common correlation axis (Z-axis).

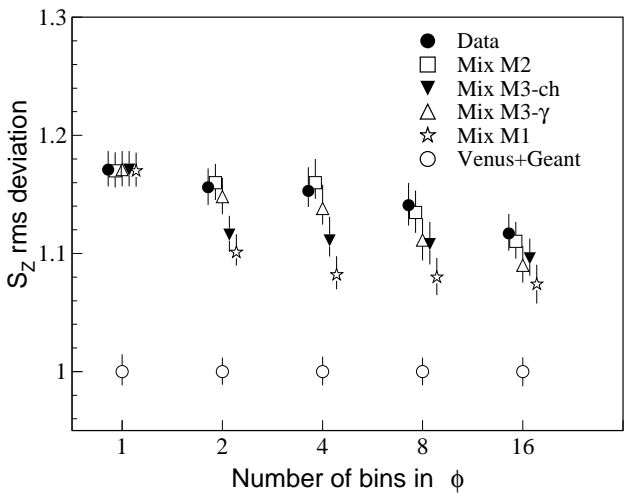

Figure 4. The root mean square (RMS) deviations of the $S_{Z}$ distribution for various divisions in the azimuthal angle. Errors include both statistical and systematic sources. 


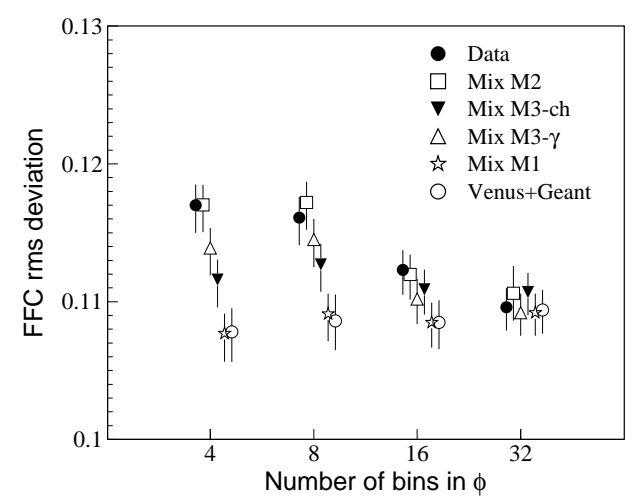

Figure 5. The root mean square (RMS) deviations of the FFC distribution for various divisions in the azimuthal angle. Errors are due to both statistical and systematic sources.

\subsection{Multi-resolution analysis based on discrete wavelet transformation}

A multi-resolution analysis using discrete wavelet transformations (DWT) has been shown to be quite powerful in the search for localized domains of DCC [12]. For the present DWT analysis the full azimuthal region is divided into smaller bins in $\phi$, the number of bins at a given scale $j$ being $2^{j}$. The input to the analysis is a spectrum of the sample function at the smallest bin in $\phi$ corresponding to the highest resolution scale, $j_{\max }(=5$ here). In the present case, the sample function is chosen to be the photon fraction, given as,

$$
f^{\prime}(\phi)=N_{\gamma-\text { like }}(\phi) /\left(N_{\gamma-\text { like }}(\phi)+N_{\mathrm{ch}}(\phi)\right)
$$

The output of the DWT consists of a set of wavelet or father function coefficients (FFC) at each scale, from $j=1, \ldots,\left(j_{\max }-1\right)$. The coefficients obtained at a given scale, $j$, are derived from the distribution of the sample function at one higher scale, $j+1$. The FFCs quantify the deviation of the bin-to-bin fluctuations in the sample function at that higher scale relative to the average behavior. The presence of localized non-statistical fluctuations will increase the RMS deviation of the distribution of FFCs and may result in nonGaussian tails 12. Once again, comparing the RMS deviations of the FFC distributions of data, mixed events, and VENUS events may allow to draw inference about the presence of localized fluctuations.

The RMS deviations of these FFC distributions are summarized in Fig. 5. The RMS deviations of the FFC distributions for the data, VENUS, and mixed events are found to be close to each other (within quoted errors) for the case of 32 bins in $\phi$. While the values for M2 mixed events are found to closely follow those of the data for all bins in $\phi$, the RMS deviations for the M3 mixed events lie between those of the data and M1 mixed events. These results are consistent with those obtained from the analysis of the $S_{Z}$ distributions. These observations indicate the absence of event-by-event localized correlated fluctuations (DCC-like) between $N_{\gamma-\text { like }}$ and $N_{\text {ch }}$. However, they do indicate 
the presence of localized independent fluctuations in both photon and charged particle multiplicities for intermediate bin sizes in azimuth.

\subsection{Centrality dependence of $N_{\gamma}$ vs. $N_{c h}$ fluctuations}

It is interesting to study the centrality dependence of $N_{\gamma}$ vs. $N_{c h}$ fluctuations [13]. This study has been carried out for four centrality classes corresponding to the top $5 \%, 5 \%$ - $10 \%, 15 \%-30 \%$, and $45 \%-55 \%$ of the minimum bias cross section. The correlation and DWT analysis was carried out on events from each of the centrality classes and the results in terms of RMS deviations of $S_{Z}$ and FFC distributions for data, various sets of mixed events, and simulations were compared. For all four classes, the RMS deviations of the $S_{Z}$ and FFC distributions of data and M2 mixed events agreed reasonably well with each other. However, comparison to M1 and M3 mixed events showed the presence of localised uncorrelated event-by-event non-statistical fluctuations in both photon and charged particle multiplicities. In order to quantify the strength of the total localised $N_{\gamma-\text { like }}$ and $N_{\text {ch }}$ fluctuations for various bins in $\phi$ and for different centrality classes, we define a sensitivity parameter $\chi$ as :

$$
\chi=\frac{\sqrt{\left(s^{2}-s_{1}^{2}\right)}}{s_{1}}
$$

where $s_{1}$ and $s$ correspond to the RMS deviations of the FFC distributions of the M1 mixed events and real data, respectively. The results are shown in Fig. 6 as a function of the number of bins in $\phi$ for the four different centrality classes. Qualitatively similar results are obtained when $\chi$ is calculated using the RMS deviations of the $S_{Z}$ distributions. The shaded portion indicates the region of $\chi$ where $s$ is one $\sigma$ greater than the RMS deviation FFC distributions for M1 events, where $\sigma$ is the total error on the M1 event RMS deviation. It represents the limit above which a signal is detectable. The result shows that the strength of the fluctuations decreases as the number of bins in $\phi$ increases, with a strength which decreases to below detectable level (within the quoted errors) for 16 and 32 bins. It is also observed that the strength of the signal decreases with decreasing centrality for 4 and 8 bins in azimuthal angle, although the tendency is not very strong.

\subsection{Upper Limit on DCC production at SPS}

If the amount of DCC-like fluctuations in the experimental data were large, then the RMS deviations of $S_{Z}$ and FFC distributions for data would have been larger than those of M2 events. Since this is not the case, we have extracted upper limits on the probability of DCC-like fluctuations at the $90 \%$ confidence level following the standard procedure as discussed in Ref. [11,14]. This is done within the context of the simple DCC model described in Ref. [11]. We give the upper limits for the two most central event classes $(0-5 \%$ and $5-10 \%)$. The $90 \% \mathrm{CL}$ upper limit contour has been calculated as $\chi+1.28 e_{\chi}$, where $\chi$ is calculated using Eqn.(6). Here $s_{1}$ and $s$ correspond to the RMS deviations of the FFC (or $S_{Z}$ ) distributions for M2 mixed events and real data, respectively, and $e_{\chi}$ is the error in $\chi$ from the FFC (or $S_{Z}$ ) analysis. To relate the measured upper limit on the size of the fluctuations in terms of DCC domain size and frequency of occurrence we take use the simple simulated DCC model described in Ref. [11]. The upper limit is set at that value of frequency of occurrence for a fixed DCC domain size at which the $\chi$ value from 


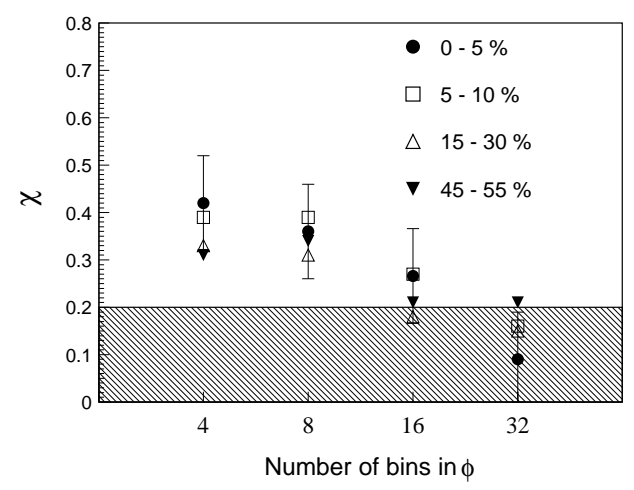

Figure 6. The fluctuation strength parameter for the four centrality classes. The error bars are shown only for the top $5 \%$ centrality class for clarity of presentation. The errors are similar for the other centralities.

the DCC model matches with that of the $\chi+1.28 e_{\chi}$ upper limit from the experimental data. The results for centrality classes 1 and 2 are shown in Fig. 0 .

\section{Summary}

A detailed study of centrality and rapidity acceptance dependence of multiplicity fluctuations carried out by the WA98 experiment at the CERN SPS shows an absence of significant non-statistical fluctuations in the photon and charged particle multiplicity. A model independent study of event-by-event correlated fluctuations (DCC-type) in photon and charged particle multiplicity using a robust mixed event technique reveals an absence of significant DCC-like correlated fluctuations at the SPS. However, the analysis indicates the presence of event-by-event uncorrelated fluctuations in both $N_{\gamma}$ and $N_{\text {ch }}$ beyond those observed in simulated and mixed events for limited region of azimuthal angle, with the strength of fluctuation increasing with increase in centrality. Using the results from the data, mixed events, and a simple model of DCC formation, an upper limit on DCC production in $\mathrm{Pb}+\mathrm{Pb}$ collisions at SPS energies has been set.

\section{Acknowledgments}

One of us (B.M) is grateful to the Board of Research on Nuclear Science and Department of Atomic Energy, Government of India for financial support in form of the Dr. K.S. Krishnan fellowship. This work was supported jointly by the German BMBF and DFG, the U.S. DOE, the Swedish NFR and FRN, the Dutch Stichting FOM, the Polish KBN the Grant Agency of the Czech Republic the DAE, DST, CSIR, UGC of the Government of India, the PPE division of CERN, the Swiss National Fund, The Ministry of Education, Science and Culture, the University of Tsukuba Special Research Projects, and the JSPS Research Fellowships for Young Scientists. 


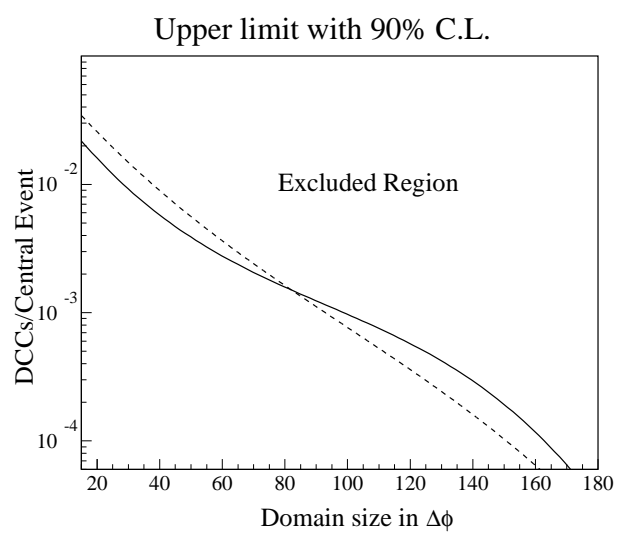

Figure 7. The $90 \%$ confidence level upper limit on $\mathrm{DCC}$ production for central $\mathrm{Pb}+\mathrm{Pb}$ collision at $158 \mathrm{~A} \mathrm{GeV} / \mathrm{c}$ as a function of the DCC domain size in azimuthal angle. The solid line corresponds to data from the top $5 \%$ and dashed line to top $5-10 \%$ of the minimum bias cross section as determined by selection on the measured transverse energy distribution.

\section{REFERENCES}

1. M. Stephanov et al., Phys. Rev. Lett. 81, 4816 (1998).

2. M. Stephanov, K. Rajagopal, E. Shuryak, Phys. Rev. D60, 114028 (1999).

3. H. Heiselberg, Phys. Rep. 351 (2001) 161 and references there in.

4. K. Rajagopal and F. Wilczek, Nucl. Phys. B399, 395 (1993).

5. H.H. Gutbrod et al., Proposal for a Large Acceptance Hadron and Photon Spectrometer, Preprint CERN/SPSLC 91-17, SPSLC/P260.

6. M.M. Aggarwal et al., (WA98 Collab.), e-print: nucl-ex/0108029.

7. M.M. Aggarwal et al., (WA98 Collab.), Phys. Lett. B458, 422 (1999).

8. M.M. Aggarwal et al., (WA98 Collab.), Phys. Lett. B420, 169 (1998).

9. D.P. Mahapatra, B. Mohanty and S.C. Phatak, Int. J. Mod. Phys. A17,675 (2002).

10. M.M. Aggarwal et al., (WA98 Collab.), Phys. Rev. C64, 011901R (2001).

11. M.M. Aggarwal et al., (WA98 Collab.), e-print : nucl-ex/0206017.

12. B.K. Nandi et al., Phys. Lett. B461 (1999) 142.

13. M. Asakawa, H. Minakata and B. Muller, Nucl. Phys. A 638, 443c (1998).

14. G.J. Feldman and R.D. Cousins, Phys. Rev. D57, 3873 (1998). 